\title{
Solvability of Fractional Differential Inclusion with a Generalized Caputo Derivative
}

\author{
Tamer Nabil $\mathbb{D}^{1,2}$ \\ ${ }^{1}$ College of Science, Department of Mathematics, King Khalid University, P.O. Box: 9004, 61413 Abha, Saudi Arabia \\ ${ }^{2}$ Faculty of Computers and Informatics, Department of Basic Science, Suez Canal University, Ismailia, Egypt \\ Correspondence should be addressed to Tamer Nabil; t_3bdelsadek@yahoo.com
}

Received 15 June 2020; Revised 28 October 2020; Accepted 20 November 2020; Published 28 December 2020

Academic Editor: Erdal Karapinar

Copyright ( 2020 Tamer Nabil. This is an open access article distributed under the Creative Commons Attribution License, which permits unrestricted use, distribution, and reproduction in any medium, provided the original work is properly cited.

This paper is devoted to the investigation of a kind of generalized Caputo semilinear fractional differential inclusions with deviatedadvanced nonlocal conditions. Solvability of the problem is established by means of the Leray-Schauder's alternative approach with the help of the Lagrange mean-value classical theorem. Finally, some examples are given to delineate the efficient of theoretical results.

\section{Introduction}

The history of the theory of fractional calculus goes back to 1695 when Leibniz sent a question to L-Hôpital [1]. Although in the starter fractional calculus had an efflorescence as a mathematical analysis idea, nowadays, its use has also sawing into many other subjects of engineering and science such as biology, physics, mechanics, chemistry, and bioengineering [2-5].

$$
\left\{\begin{array}{l}
\eta^{\prime}(t) \in F(t, \eta(t)), \quad \text { a.e. } \quad t \in(0,1) \\
\sum_{i=1}^{l_{1}} c_{i} \eta\left(\theta\left(t_{i}\right)\right)=\beta \sum_{j=1}^{l_{2}} d_{j} \eta\left(\phi\left(\tau_{i}\right)\right), \quad c_{i}, d_{j}>0, \quad t_{i}, \tau_{j} \in(0,1), \forall i=1, \cdots, l_{1}, j=1, \cdots, l_{2} .
\end{array}\right.
$$

In the few past years, there has been important works in fractional differential inclusions with other types of nonlocal conditions. Detailedly, in 2015, Wang et al. [9] established the existence of solutions for the Caputo fractional differential inclusions involving nonlocal conditions. In the second year, Lian et al. [10] established the solvability of the frac-
It is known that differential inclusions are more general than differential equations and various phenomena of science, control, and engineering are successfully modeled as fractional differential inclusions $[6,7]$.

Recently, fractional differential inclusions with nonlocal conditions have attracted the attention of many researchers. In 2011, El-Sayed et al. [8] established the solvability of the ordinary differential inclusion with deviated-advanced nonlocal condition.

tional differential inclusions with nonlocal conditions by using the measure of noncompactness and several-valued fixed-point approach. In 2019, Castaing et al. [11] studied the solvability of a new class of the Riemann-Liouville fractional differential inclusion with nonlocal integral conditions in a separable Banach space. 
In the above-cited monographs, the Caputo and Riemann-Liouville derivatives were utilized. In 2017, Almeida [12] obtained the new generalized Caputo fractional derivative, that is, a Caputo-kind operator of a function with respect to another function. Indeed, this fractional operator is more general than Riemann-Liouville, Hadamard, Erdely Kober, and Caputo operator kinds. More details about the generalized Caputo fractional operator are found in $[13,14]$. Since then, generalized fractional operators draw increasing attention due to their advantages, because the generalized fractional operators will give us new opportunities to improve the theoretical results and to model a lot of real-life events. In 2019, Promsakon et al. [15] established the solvability of a new class of impulsive fractional boundary value problems involving the generalized Caputo fractional derivative. In 2020, Belmor et al. [16] investigated the solvability of fractional differential inclusion including the generalized Caputo derivative with integral nonlocal conditions. There are other works that showed interest in the generalized Caputo operators; we mention for example [17-20].

Nowadays, Herzallah and Radwan [21] studied the fractional version of the system (1) with the classical Caputo operator, namely

$$
\left\{\begin{array}{l}
D_{0+}^{\alpha} \eta(t) \in A(t) \eta(t)+F(t, \eta(t)), \quad \text { a.e. } \quad t \in[0, T], T>0, \\
\sum_{i=1}^{l_{1}} c_{i} \eta\left(\theta\left(t_{i}\right)\right)=\beta \sum_{j=1}^{l_{2}} d_{j} \eta\left(\phi\left(\tau_{i}\right)\right), c_{i}, d_{j}>0, t_{i}, \tau_{j} \in(0, T), \forall i=1, \cdots, l_{1}, j=1, \cdots, l_{2} .
\end{array}\right.
$$

Motivated by the above-cited contributions, in particular systems (1) and (2), we propose a new fractional differential inclusion involving generalized Caputo operator, given by

$$
\left\{\begin{array}{l}
D_{0^{+}, Q}^{\alpha} \eta(t) \in A(t) \eta(t)+F(t, \eta(t)), \quad \text { a.e. } \quad t \in I=[0, T], T>0, \\
\sum_{i=1}^{l_{1}} c_{i} \eta\left(\theta\left(t_{i}\right)\right)=\beta \sum_{j=1}^{l_{2}} d_{j} \eta\left(\phi\left(\tau_{i}\right)\right), c_{i}, d_{j}>0, t_{i}, \tau_{j} \in I, \forall i=1, \cdots, l_{1}, j=1, \cdots, l_{2} .
\end{array}\right.
$$

where ${ }^{*} D_{0^{+}, Q}^{\alpha}$ is the generalized Caputo derivative w.r.t. the function $Q$ such that $\alpha \in(0,1), A(t): D(A) \subseteq \mathbb{R} \longrightarrow \mathbb{R}$ is linear bounded operator and $F: I \times \mathbb{R} \longrightarrow P\left(\mathbb{R}_{+}\right)$. We show the existence of solution for the proposed system (3). The proposed system (3) is more flexible since it allows us to choose fractional derivative depending on the particular established phenomenon. Therefore, the tools of generalized fractional differential inclusions facilitate the investigation of optimal controls and stochastic processing, in particular, modeling of control processes that are considered by selecting a trial function [7]. Moreover, nonlocal conditions give more accurate measurements, precise results, and efficient effect than the classical boundary conditions.

An outline of this paper is as follows. In Section 2, some bases and results are given needed in the sequel. In Section 3 , we study the solvability of the generalized system (3). In Section 4, we apply the abstract results in order to establish the existence of solution for some illustrative examples.

\section{Preliminaries}

In this part, we recall some definitions and theorems that will be used later. Let $\left(E,\|.\|_{E}\right)$ be a Banach space and $P$
$(E)=\{Z \subset E: Z \neq \varnothing\}$. Now, throughout this paper, let

$$
\begin{gathered}
P_{b d}(E)=\{Z \in P(E): Z \text { is bounded }\}, \\
P_{c p}(E)=\{Z \in P(E): Z \text { is compact }\}, \\
P_{c v}(E)=\{Z \in P(E): Z \text { is convex }\}, \\
P_{c v p}(E)=P_{c p}(E) \cap P_{c v}(E) .
\end{gathered}
$$

Let $W \subseteq E$. The fixed point of set-valued map $\Psi: W$ $\longrightarrow P(E)$ is a point $\omega \in W$ such that $\omega \in \Psi(\omega)$. The graph of $\Psi$ is defined as

$$
G(\Psi)=\left\{\left(\omega_{1}, \omega_{2}\right) \in W \times E: \omega_{2} \in \Psi\left(w_{1}\right)\right\}
$$

A selection of $\Psi$ is a single-valued map $\psi: W \longrightarrow E$ such that $G(\psi) \subseteq G(\Psi)$.

$\Psi: E \longrightarrow P(E)$ is closed (convex) valued if $\Psi(\omega)$ is closed (convex) for each $\omega \in E$, and $\Psi$ is bounded on bounded sets if $\Psi(W)=\bigcup_{\omega \in W} \Psi(\omega)$ is bounded for each $W$ $\in P_{b d}(E)$, that is, $\sup _{\omega \in W}\{\sup \{\|z\|: z \in \Psi(\omega)\}\}<\infty$.

Therefore, $\Psi$ is completely continuous if $\Psi(W)$ is relatively compact for each $W \in P_{b d}(W)$. In fact, if $\Psi$ is completely continuous with nonempty compact values, then $\Psi$ is upper semicontinuous (u.s.c., for short) if and only if $G$ $(\Psi)$ is closed.

Let $L^{1}(I, E)=\left\{\eta: I \longrightarrow E:\|\eta\|_{E}: I \longrightarrow R_{+}\right.$be Lebesgue integrable $\}$; then $L^{1}(I, E)$ is Banach with the norm $\|\eta\|_{L^{1}}=$ $\int_{I}\|\eta(t)\|_{E} d t$.

Definition 1 [22]. A multivalued function $\Psi: I \times E \longrightarrow P(E)$ is called $L^{1}$-Carathéodory if

(i) $t \longrightarrow \Psi(t, \omega)$ is measurable for each $\omega \in E$,

(ii) $\omega \longrightarrow \Psi(t, \omega)$ is u.s.c. for almost all $t \in I$, 
(iii) for each $r \in(0, \infty)$, there exists $p_{r} \in L^{1}\left(I, R_{+}\right)$such that

$\|\Psi(t, \omega)\|=\sup \left\{\|v\|_{E}: v \in \Psi(t, \omega)\right\} \leq p_{r}(t), \forall\|\omega\|_{E} \leq r$ and almost every $t \in I$.

Definition 2 [23]. Let $C(I, E)$ be the Banach space of all continuous functions $\eta: I \longrightarrow E$ with the norm $\|\eta\|_{\infty}=\sup _{t \in I}$ $\|\eta(t)\|_{E}$. Therefore, let $C^{m}(I, E)$ be the Banach space of all $m$-differentiable maps $\eta: I \longrightarrow E$ with $\eta^{(m)}(t) \in C(I, E), m$ $\in \mathbb{N}$.

Definition 3 [24]. For every $\eta \in C(I, E)$, define the family of $L^{1}$ -selection of $\Psi: I \times E \longrightarrow P_{c v p}(E)$ as

$$
S_{\Psi, \eta}^{1}=\left\{\psi \in L^{1}(I, \mathbb{R}): \psi(t) \in \Psi(t, \eta(t)) \text { for a.e.t } \in I\right\} \text {. }
$$

Therefore, $S_{\Psi, \eta}^{1}$ is a nonempty set.

Lemma 4 [24]. Let $\Psi: I \times E \longrightarrow P_{c v p}(E)$ be a $L^{1}-$ Carathéodory-multivalued function and $\Phi: L^{1}(I, E) \longrightarrow C(I, E)$ be a continuous linear mapping. Then

$$
\Phi \circ S_{\Psi}^{1}: C(I, E) \longrightarrow P_{c v p}(C(I, E)), \eta \mapsto \Phi\left(S_{\Psi, \eta}^{1}\right)
$$

is a closed graph operator in $C(I, E) \times C(I, E)$.

An important role is played by the fixed-point principle to obtain the solvability of various types of operator equations (see, for example, [25-29]). We will apply the following fixed-point theorem to obtain the main results.

Theorem 5 [30]. Let $Z$ be a convex closed subset of $E, \Omega$ be an open subset of $Z, 0 \in \Omega$ and $\Psi: \bar{\Omega} \longrightarrow P_{c v p}(Z)$ is u.s.c. and compact operator. Then, either

(1) $\Psi$ has a fixed point in $\bar{\Omega}$, or

(2) there exists $\omega \in \partial \Omega$ and $\lambda \in(0,1)$ with $\omega \in \lambda \Psi \omega$.

Next, we outline some definitions of the generalized fractional operators $[12,13]$. For more details about fractional operators, the readers are also referred to $[1,31]$.

Definition 6. Let $Q:(a, b) \longrightarrow \mathbb{R}_{+}$be an increasing function having a derivative $Q^{\prime}(t) \in C((a, b), \mathbb{R})$ such that $Q^{\prime}(t) \neq 0$ for all $t \in(a, b)$. The left generalized Riemann-Liouville fractional integral of order $\alpha \in(n, n+1)$ for some $n \in \mathbb{N}$ of an integrable function $\eta:[a, b] \longrightarrow \mathbb{R}$ w.r.t. the function $Q$ is given by [12]

$$
J_{a^{+}, Q}^{\alpha} \eta(t)=\frac{1}{\Gamma(\alpha)} \int_{a}^{t} Q^{\prime}(\zeta)(Q(t)-Q(\zeta))^{\alpha-1} \eta(\zeta) d \zeta, \quad t>a .
$$

Choosing $Q(t)=\ln (t)$ and replacing in (8), we have the
Hadamard fractional integral, given by [32]

$$
{ }^{H} J_{a^{+}}^{\alpha} \eta(t)=\frac{1}{\Gamma(\alpha)} \int_{a}^{t}\left(\ln \left(\frac{t}{\zeta}\right)\right)^{\alpha-1} \eta(\zeta) \frac{d \zeta}{\zeta}, \quad a>0, t>a
$$

Choosing $Q(t)=t$ and replacing in (8), we get the classical Riemann-Liouville integral, given by [1]

$$
J_{a^{+}}^{\alpha} \eta(t)=\frac{1}{\Gamma(\alpha)} \int_{a}^{t}(t-\zeta)^{\alpha-1} \eta(\zeta) d \zeta, \quad t>a .
$$

The left generalized Riemann-Liouville fractional derivative of order $\alpha \in(n, n+1)$ for some $n \in \mathbb{N}$ of an integrable function $\eta:[a, b] \longrightarrow \mathbb{R}$ w.r.t. the function $Q$ is given by [12]

$$
\begin{aligned}
D_{a^{+}, Q}^{\alpha} \eta(t)= & \frac{1}{\Gamma(n-\alpha)}\left(\frac{1}{Q^{\prime}(t)} \frac{d}{d t}\right)^{n} \int_{a}^{t} Q^{\prime}(\zeta) \\
& \cdot(Q(t)-Q(\zeta))^{n-\alpha-1} \eta(\zeta) d \zeta \quad t>a .
\end{aligned}
$$

Choosing $Q(t)=\ln (t)$ and replacing in (11), we have the Hadamard fractional derivative, given by [32]

$$
{ }^{H} D_{a^{+}}^{\alpha} \eta(t)=\frac{1}{\Gamma(n-\alpha)}\left(t \frac{d}{d t}\right)^{n} \int_{a}^{t}\left(\ln \left(\frac{t}{\zeta}\right)\right)^{n-\alpha-1} \eta(\zeta) d \zeta, \quad a>0, t>a .
$$

Choosing $Q(t)=t$ and replacing in (11), we get classical Riemann-Liouville derivative, given by [31]

$$
D_{a^{+}}^{\alpha} \eta(t)=\frac{1}{\Gamma(n-\alpha)}\left(\frac{d}{d t}\right)^{n} \int_{a}^{t}(t-\zeta)^{n-\alpha-1} \eta(\zeta) d \zeta, \quad t>a .
$$

Definition 7. Let $\alpha \in(n, n+1)$ for some $n \in \mathbb{N}$ and $Q \in C^{n}$ $((a, b), \mathbb{R})$ be an increasing mapping such that $Q^{\prime}(t) \neq 0$ for all $t \in(a, b)$. Consider $\eta:(a, b) \longrightarrow \mathbb{R}$ be an integrable function. The left generalized Caputo fractional derivative of order $\alpha>0, \alpha \in \mathbb{R}_{+}$, w.r.t. the function $\mathrm{Q}$ is given by [12]

$$
{ }^{*} D_{a^{+}, Q}^{\alpha} \eta(t)=J_{a^{+}, Q}^{n-\alpha}\left(\frac{1}{Q^{\prime}(t)} \frac{d}{d t}\right)^{n} \eta(t), \quad t>a .
$$

Choosing $Q(t)=t$ and replacing in (14), we obtain the classical Caputo fractional derivative. Choosing $Q(t)=\ln$ $(t)$ and replacing in (14), we have the Caputo-Hadamard fractional derivative, given by [32]

$$
\begin{aligned}
{ }^{C-H} D_{a^{+}}^{\alpha} \eta(t) & =\frac{1}{\Gamma(n-\alpha)} \int_{a}^{t}\left(\ln \frac{\zeta}{t}\right)^{n-\alpha-1}\left(t \frac{d}{d t}\right)^{n} \eta(t) \frac{d t}{t} \\
& =\left({ }^{H} J_{a^{+}}^{n-\alpha}\left(t \frac{d}{d t}\right)^{n} \eta(t)\right), \quad a>0, t>a .
\end{aligned}
$$


Further, the generalized Caputo derivative can be defined via the generalized Riemann-Liouville fractional derivative as [13]

$$
{ }^{*} D_{a^{+}, Q}^{\alpha} \eta(t)=D_{a^{+}, Q}^{\alpha}\left[\eta(t)-\sum_{k=0}^{n-1} \frac{\eta_{Q}^{[k]}(a)}{k !}(Q(t)-Q(a))^{k}\right],
$$

where $\eta_{Q}^{[k]}(t)=\left(\left(1 / Q^{\prime}(t)\right)(d / d t)\right)^{k} \eta(t)$.

The following lemma, which concerns some properties of generalized fractional operators, plays a key role in the sequel.

Lemma 8. [13]. Suppose that $\eta: I \longrightarrow \mathbb{R}$, then

(1) if $\eta \in C(I, \mathbb{R})$, then ${ }^{*} D_{0^{+}, Q}^{\alpha} J_{0^{+}, Q}^{\alpha} \eta(t)=\eta(t)$,

(2) if $\eta \in C^{n}(I, \mathbb{R})$, then

$$
J_{0^{+}, Q}^{\alpha}{ }^{*} D_{0^{+}, Q}^{\alpha} \eta(t)=\eta(t)-\sum_{k=0}^{n-1} \frac{\eta_{Q}^{[k]}(0)}{k !}(Q(t)-Q(0))^{k}
$$

(3) if $\eta \in C^{n}(I, \mathbb{R})$ and $\alpha \in(0,1)$, then

$$
J_{0^{+}, Q}^{\alpha}{ }^{*} D_{0^{+}, Q}^{\alpha} \eta(t)=\eta(t)-\eta(0) .
$$

\section{Main Results}

The differential inclusions using fractional derivatives have been proven to be of major interest to the academic community, not only mathematicians but also researchers in other fields. There is a motivating way to obtain the solvability of the differential inclusions; this way is representing the solution by integral equation.

The solvability of system (3) will be established under the following hypotheses:

(H1) For all $\eta(t) \in I$, there exists $L_{A} \in(0, \infty)$ such that $L_{A}=\max _{t \in I}|A(t)|$.

(H2) $\sum_{i=1}^{l_{1}} c_{i} \neq \beta \sum_{j=1}^{l_{2}} d_{j}$.

(H3) The function $F: I \times \mathbb{R} \longrightarrow \mathbb{R}$ is $L^{1}$-Carathéodory and has nonempty, convex, and compact values.

(H4) The functions $\theta, \phi: I \longrightarrow I$ are continuous such that $\theta(t) \leq t$ and $\phi(t) \geq t$ for all $t \in I$.

(H5) There exists a function $p \in L^{1}\left(I, R_{+}\right)$and $K>0$ such that $\|F(t, \eta(t))\| \leq K p(t)$, and there exists $M^{*}>0$ such that

$\frac{\Gamma(\alpha+1) M^{*}}{\left(L_{A} M^{*}(Q(T)-Q(0))^{\alpha}+K M \Gamma(\alpha+1)\right)\left(|\sigma \beta| \sum_{j=1}^{l_{2}} d_{j}+|\sigma| \sum_{i=1}^{l_{1}} c_{i}+1\right)}>1$, where

$$
M=\frac{1}{\Gamma(\alpha)} \int_{0}^{T} Q^{\prime}(s)(Q(T)-Q(s))^{\alpha-1} p(s) d s .
$$

The integral representation of the system (3) will be given in the following lemma.

Lemma 9. Let the hypotheses (H1)-(H2) hold. Suppose that $\psi: I \longrightarrow C(I, \mathbb{R})$, then the solution $\eta(t)$ of the following problem

$\left\{\begin{array}{l}{ }^{*} D_{0^{+}, Q}^{\alpha} \eta(t)=A(t) \eta(t)+\psi(t), \quad \text { a.e. } \quad t \in I, \\ \sum_{i=1}^{l_{l}} c_{i} \eta\left(\theta\left(t_{i}\right)\right)=\beta \sum_{j=1}^{l_{2}} d_{j} \eta\left(\phi\left(\tau_{i}\right)\right), \quad c_{i}, d_{j}>0, \quad t_{i}, \tau_{j} \in I, \forall i=1, \cdots, l_{1}, j=1, \cdots l_{2},\end{array}\right.$

is given by

$$
\begin{aligned}
\eta(t)= & \sigma \beta \sum_{j=1}^{l_{2}} d_{j} \int_{0}^{\phi\left(\tau_{j}\right)} \frac{Q^{\prime}(s)\left(Q\left(\phi\left(\tau_{j}\right)\right)-Q(s)\right)^{\alpha-1}}{\Gamma(\alpha)} A(s) \eta(s) d s \\
& +\sigma \beta \sum_{j=1}^{l_{2}} d_{j} \int_{0}^{\phi\left(\tau_{j}\right)} \frac{Q^{\prime}(s)\left(Q\left(\phi\left(\tau_{j}\right)\right)-Q(s)\right)^{\alpha-1}}{\Gamma(\alpha)} \psi(s) d s \\
& -\sigma \sum_{i=1}^{l_{1}} c_{i} \int_{0}^{\theta\left(t_{i}\right)} \frac{Q^{\prime}(s)\left(Q\left(\theta\left(t_{i}\right)\right)-Q(s)\right)^{\alpha-1}}{\Gamma(\alpha)} A(s) \eta(s) d s \\
& -\sigma \sum_{i=1}^{l_{1}} c_{i} \int_{0}^{\theta\left(t_{i}\right)} \frac{Q^{\prime}(s)\left(Q\left(\theta\left(t_{i}\right)\right)-Q(s)\right)^{\alpha-1}}{\Gamma(\alpha)} \psi(s) d s \\
& +\frac{1}{\Gamma(\alpha)} \int_{0}^{t} Q^{\prime}(s)(Q(t)-Q(s))^{\alpha-1} A(s) \eta(s) d s \\
& +\frac{1}{\Gamma(\alpha)} \int_{0}^{t} Q^{\prime}(s)(Q(t)-Q(s))^{\alpha-1} \psi(s) d s,
\end{aligned}
$$

where $\sigma=1 /\left(\sum_{i=1}^{l_{1}} c_{i}-\beta \sum_{j=1}^{l_{2}} d_{j}\right)$.

Proof. Applying the operator $J_{0^{+}, Q}^{\alpha}$ on both sides of equation (21). Then, by Lemma 8, we get

$$
J_{0^{+}, Q}^{\alpha}{ }^{*} D_{0^{+}, Q}^{\alpha} \eta(t)=J_{0^{+}, Q}^{\alpha}[A(t) \eta(t)+\psi(t)] .
$$

Therefore, we obtain

$$
\begin{aligned}
\eta(t)= & \eta(0)+\frac{1}{\Gamma(\alpha)} \int_{0}^{t} Q^{\prime}(s)(Q(t)-Q(s))^{\alpha-1} A(s) \eta(s) d s \\
& +\frac{1}{\Gamma(\alpha)} \int_{0}^{t} Q^{\prime}(s)(Q(t)-Q(s))^{\alpha-1} \psi(s) d s .
\end{aligned}
$$


Putting $t=\theta\left(t_{i}\right)$ in equation (24), we get

$$
\begin{aligned}
\eta\left(\theta\left(t_{i}\right)\right)= & \eta(0)+\frac{1}{\Gamma(\alpha)} \int_{0}^{\theta\left(t_{i}\right)} Q^{\prime}(s)\left(Q\left(\theta\left(t_{i}\right)\right)-Q(s)\right)^{\alpha-1} A(s) \eta(s) d s \\
& +\frac{1}{\Gamma(\alpha)} \int_{0}^{\theta\left(t_{i}\right)} Q^{\prime}(s)\left(Q\left(\theta\left(t_{i}\right)\right)-Q(s)\right)^{\alpha-1} \psi(s) d s .
\end{aligned}
$$

Thus, we have

$$
\begin{aligned}
\sum_{i=1}^{l_{1}} c_{i} \eta\left(\theta\left(t_{i}\right)\right)= & \sum_{i=1}^{l_{i}} c_{i} \eta(0)+\sum_{i=1}^{l_{1}} c_{i} \frac{1}{\Gamma(\alpha)} \int_{0}^{\theta\left(t_{i}\right)} Q^{\prime}(s)\left(Q\left(\theta\left(t_{i}\right)\right)\right. \\
& -Q(s))^{\alpha-1} A(s) \eta(s) d s+\sum_{i=1}^{l_{1}} c_{i} \frac{1}{\Gamma(\alpha)} \int_{0}^{\theta\left(t_{i}\right)} Q^{\prime}(s) \\
& \cdot\left(Q\left(\theta\left(t_{i}\right)\right)-Q(s)\right)^{\alpha-1} \psi(s) d s .
\end{aligned}
$$

Putting $t=\phi\left(\tau_{j}\right)$ in equation (24), we get

$$
\begin{aligned}
\eta\left(\phi\left(\tau_{j}\right)\right)= & \eta(0)+\frac{1}{\Gamma(\alpha)} \int_{0}^{\phi\left(\tau_{j}\right)} Q^{\prime}(s) \\
& \cdot\left(Q\left(\phi\left(\tau_{j}\right)\right)-Q(s)\right)^{\alpha-1} A(s) \eta(s) d s \\
& +\frac{1}{\Gamma(\alpha)} \int_{0}^{\phi\left(\tau_{j}\right)} Q^{\prime}(s)\left(Q\left(\phi\left(\tau_{j}\right)\right)-Q(s)\right)^{\alpha-1} \psi(s) d s .
\end{aligned}
$$

Thus, we have

$$
\begin{aligned}
\sum_{j=1}^{l_{2}} d_{j} \eta\left(\phi\left(\tau_{j}\right)\right)= & \sum_{j=1}^{l_{2}} d_{j} \eta(0)+\sum_{j=1}^{l_{2}} d_{j} \frac{1}{\Gamma(\alpha)} \int_{0}^{\phi\left(\tau_{j}\right)} Q^{\prime}(s) \\
& \cdot\left(Q\left(\phi\left(\tau_{j}\right)\right)-Q(s)\right)^{\alpha-1} A(s) \eta(s) d s \\
& +\sum_{j=1}^{l_{2}} d_{j} \frac{1}{\Gamma(\alpha)} \int_{0}^{\phi\left(\tau_{j}\right)} Q^{\prime}(s) \\
& \cdot\left(Q\left(\phi\left(\tau_{j}\right)\right)-Q(s)\right)^{\alpha-1} \psi(s) d s .
\end{aligned}
$$

Hence, we obtain

$$
\begin{aligned}
\eta(0)= & \sigma \beta \sum_{j=1}^{l_{2}} d_{j} \int_{0}^{\phi\left(\tau_{j}\right)} \frac{Q^{\prime}(s)\left(Q\left(\phi\left(\tau_{j}\right)\right)-Q(s)\right)^{\alpha-1}}{\Gamma(\alpha)} A(s) \eta(s) d s \\
& +\sigma \beta \sum_{j=1}^{l_{2}} d_{j} \int_{0}^{\phi\left(\tau_{j}\right)} \frac{Q^{\prime}(s)\left(Q\left(\phi\left(\tau_{j}\right)\right)-Q(s)\right)^{\alpha-1}}{\Gamma(\alpha)} \psi(s) d s \\
& -\sigma \sum_{i=1}^{l_{1}} c_{i} \int_{0}^{\theta\left(t_{i}\right)} \frac{Q^{\prime}(s)\left(Q\left(\theta\left(t_{i}\right)\right)-Q(s)\right)^{\alpha-1}}{\Gamma(\alpha)} A(s) \eta(s) d s \\
& -\sigma \sum_{i=1}^{l_{1}} c_{i} \int_{0}^{\theta\left(t_{i}\right)} \frac{Q^{\prime}(s)\left(Q\left(\theta\left(t_{i}\right)\right)-Q(s)\right)^{\alpha-1}}{\Gamma(\alpha)} \psi(s) d s .
\end{aligned}
$$

Substituting equation (29) into equation (24), we obtain the result.

We note that a function $\eta \in C(I, \mathbb{R})$ is called a solution for system (3) if there exists a map $\psi \in L^{1}(I, \mathbb{R})$ such that $\psi \in F$ $(t, \eta(t))$ a.e. on $I$ and $\eta(t)$ is given by

$$
\begin{aligned}
\eta(t)= & \sigma \beta \sum_{j=1}^{l_{2}} d_{j} \int_{0}^{\phi\left(\tau_{j}\right)} \frac{Q^{\prime}(s)\left(Q\left(\phi\left(\tau_{j}\right)\right)-Q(s)\right)^{\alpha-1}}{\Gamma(\alpha)} A(s) \eta(s) d s \\
& +\sigma \beta \sum_{j=1}^{l_{2}} d_{j} \int_{0}^{\phi\left(\tau_{j}\right)} \frac{Q^{\prime}(s)\left(Q\left(\phi\left(\tau_{j}\right)\right)-Q(s)\right)^{\alpha-1}}{\Gamma(\alpha)} \psi(s) d s \\
& -\sigma \sum_{i=1}^{l_{1}} c_{i} \int_{0}^{\theta\left(t_{i}\right)} \frac{Q^{\prime}(s)\left(Q\left(\theta\left(t_{i}\right)\right)-Q(s)\right)^{\alpha-1}}{\Gamma(\alpha)} A(s) \eta(s) d s \\
& -\sigma \sum_{i=1}^{l_{1}} c_{i} \int_{0}^{\theta\left(t_{i}\right)} \frac{Q^{\prime}(s)\left(Q\left(\theta\left(t_{i}\right)\right)-Q(s)\right)^{\alpha-1}}{\Gamma(\alpha)} \psi(s) d s \\
& +\frac{1}{\Gamma(\alpha)} \int_{0}^{t} Q^{\prime}(s)(Q(t)-Q(s))^{\alpha-1} A(s) \eta(s) d s \\
& +\frac{1}{\Gamma(\alpha)} \int_{0}^{t} Q^{\prime}(s)(Q(t)-Q(s))^{\alpha-1} \psi(s) d s,
\end{aligned}
$$

where $\sigma=1 /\left(\sum_{i=1}^{l_{1}} c_{i}-\beta \sum_{j=1}^{l_{2}} d_{j}\right)$.

Now, we establish the solvability of problem (3).

Theorem 10. Suppose that the hypotheses (H1)-(H5) are satisfied; then the system (3) has at least one solution.

Proof. By hypothesis (H3) and Lemma 4, there exists a singlevalue map $\psi \in S_{F, \eta}^{1}$. Define the multivalued operator $r: C($ $I, \mathbb{R}) \longrightarrow P(C(I, \mathbb{R}))$ as

$$
\begin{aligned}
\Upsilon_{\eta}(t)=\{ & \{h \in C(I, \mathbb{R}): h(t) \\
= & \left\{\sigma \beta \sum_{j=1}^{L_{2}} d_{j} \int_{0}^{\phi\left(\tau_{j}\right)} \frac{Q^{\prime}(s)\left(Q\left(\phi\left(\tau_{j}\right)\right)-Q(s)\right)^{\alpha-1}}{\Gamma(\alpha)} A(s) \eta(s) d s\right. \\
& +\sigma \beta \sum_{j=1}^{L_{2}} d_{j} \int_{0}^{\phi\left(\tau_{j}\right)} \frac{Q^{\prime}(s)\left(Q\left(\phi\left(\tau_{j}\right)\right)-Q(s)\right)^{\alpha-1}}{\Gamma(\alpha)} \psi(s) d s \\
& -\sigma \sum_{i=1}^{l_{1}} c_{j} \int_{0}^{\theta\left(t_{i}\right)} \frac{Q^{\prime}(s)\left(Q\left(\theta\left(t_{i}\right)\right)-Q(s)\right)^{\alpha-1}}{\Gamma(\alpha)} A(s) \eta(s) d s \\
& -\sigma \sum_{i=1}^{l_{1}} c_{j} \int_{0}^{\theta\left(t_{i}\right)} \frac{Q^{\prime}(s)\left(Q\left(\theta\left(t_{i}\right)\right)-Q(s)\right)^{\alpha-1}}{\Gamma(\alpha)} \psi(s) d s \\
& +\int_{0}^{t} \frac{Q^{\prime}(s)(Q(t)-Q(s))^{\alpha-1}}{\Gamma(\alpha)} A(s) \eta(s) d s \\
& \left.+\int_{0}^{t} \frac{Q^{\prime}(s)(Q(t)-Q(s))^{\alpha-1}}{\Gamma(\alpha)} \psi(s) d s, \quad \psi \in S_{F, \eta}^{1}\right\} .
\end{aligned}
$$

Then for every $h \in \Upsilon_{\eta}$ and $\eta \in C(I, \mathbb{R})$, there exists $\psi \epsilon$ $S_{F, \eta}^{1}$ such that 


$$
\begin{aligned}
h(t)= & \sigma \beta \sum_{j=1}^{l_{2}} d_{j} \int_{0}^{\phi\left(\tau_{j}\right)} \frac{Q^{\prime}(s)\left(Q\left(\phi\left(\tau_{j}\right)\right)-Q(s)\right)^{\alpha-1}}{\Gamma(\alpha)} A(s) \eta(s) d s \\
& +\sigma \beta \sum_{j=1}^{l_{2}} d_{j} \int_{0}^{\phi\left(\tau_{j}\right)} \frac{Q^{\prime}(s)\left(Q\left(\phi\left(\tau_{j}\right)\right)-Q(s)\right)^{\alpha-1}}{\Gamma(\alpha)} \psi(s) d s \\
& -\sigma \sum_{i=1}^{l_{1}} c_{i} \int_{0}^{\theta\left(t_{i}\right)} \frac{Q^{\prime}(s)\left(Q\left(\theta\left(t_{i}\right)\right)-Q(s)\right)^{\alpha-1}}{\Gamma(\alpha)} A(s) \eta(s) d s \\
& -\sigma \sum_{i=1}^{l_{1}} c_{i} \int_{0}^{\theta\left(t_{i}\right)} \frac{Q^{\prime}(s)\left(Q\left(\theta\left(t_{i}\right)\right)-Q(s)\right)^{\alpha-1}}{\Gamma(\alpha)} \psi(s) d s \\
& +\frac{1}{\Gamma(\alpha)} \int_{0}^{t} Q^{\prime}(s)(Q(t)-Q(s))^{\alpha-1} A(s) \eta(s) d s \\
& +\frac{1}{\Gamma(\alpha)} \int_{0}^{t} Q^{\prime}(s)(Q(t)-Q(s))^{\alpha-1} \psi(s) d s .
\end{aligned}
$$

Now, we can obtain the proof in 4 steps.

Step 1. $\Upsilon_{\eta}$ is convex for all $\eta \in C(I, \mathbb{R})$. Let $h_{1}, h_{2} \in \Upsilon_{\eta}$; then there exists $\psi_{1}, \psi_{2} \in S_{F, \eta}^{1}$ such that for every $t \in I$, we get

$$
\begin{aligned}
h_{1}(t)= & \sigma \beta \sum_{j=1}^{l_{2}} d_{j} \int_{0}^{\phi\left(\tau_{j}\right)} \frac{Q^{\prime}(s)\left(Q\left(\phi\left(\tau_{j}\right)\right)-Q(s)\right)^{\alpha-1}}{\Gamma(\alpha)} A(s) \eta(s) d s \\
& +\sigma \beta \sum_{j=1}^{l_{2}} d_{j} \int_{0}^{\phi\left(\tau_{j}\right)} \frac{Q^{\prime}(s)\left(Q\left(\phi\left(\tau_{j}\right)\right)-Q(s)\right)^{\alpha-1}}{\Gamma(\alpha)} \psi_{1}(s) d s \\
& -\sigma \sum_{i=1}^{l_{1}} c_{i} \int_{0}^{\theta\left(t_{i}\right)} \frac{Q^{\prime}(s)\left(Q\left(\theta\left(t_{i}\right)\right)-Q(s)\right)^{\alpha-1}}{\Gamma(\alpha)} A(s) \eta(s) d s \\
& -\sigma \sum_{i=1}^{l_{1}} c_{i} \int_{0}^{\theta\left(t_{i}\right)} \frac{Q^{\prime}(s)\left(Q\left(\theta\left(t_{i}\right)\right)-Q(s)\right)^{\alpha-1}}{\Gamma(\alpha)} \psi_{1}(s) d s \\
& +\frac{1}{\Gamma(\alpha)} \int_{0}^{t} Q^{\prime}(s)(Q(t)-Q(s))^{\alpha-1} A(s) \eta(s) d s \\
& +\frac{1}{\Gamma(\alpha)} \int_{0}^{t} Q^{\prime}(s)(Q(t)-Q(s))^{\alpha-1} \psi_{1}(s) d s, \\
h_{2}(t)= & \sigma \beta \sum_{j=1}^{l_{2}} d_{j} \int_{0}^{\phi\left(\tau_{j}\right)} \frac{Q^{\prime}(s)\left(Q\left(\phi\left(\tau_{j}\right)\right)-Q(s)\right)^{\alpha-1}}{\Gamma(\alpha)} A(s) \eta(s) d s \\
& +\sigma \beta \sum_{j=1}^{l_{2}} d_{j} \int_{0}^{\phi\left(\tau_{j}\right)} \frac{Q^{\prime}(s)\left(Q\left(\phi\left(\tau_{j}\right)\right)-Q(s)\right)^{\alpha-1}}{\Gamma(\alpha)} \psi_{2}(s) d s \\
& -\sigma \sum_{i=1}^{l_{1}} c_{i} \int_{0}^{\theta\left(t_{i}\right)} \frac{Q^{\prime}(s)\left(Q\left(\theta\left(t_{i}\right)\right)-Q(s)\right)^{\alpha-1}}{\Gamma(\alpha)} A(s) \eta(s) d s \\
& -\sigma \sum_{i=1}^{l_{1}} c_{i} \int_{0}^{\theta\left(t_{i}\right)} \frac{Q^{\prime}(s)\left(Q\left(\theta\left(t_{i}\right)\right)-Q(s)\right)^{\alpha-1}}{\Gamma(\alpha)} \psi_{2}(s) d s \\
& +\frac{1}{\Gamma(\alpha)} \int_{0}^{t} Q^{\prime}(s)(Q(t)-Q-(s))^{\alpha-1} A(s) \eta(s) d s \\
& +\frac{1}{\Gamma(\alpha)} \int_{0}^{t} Q^{\prime}(s)(Q(t)-Q-(s))^{\alpha-1} \psi_{2}(s) d s . \\
&
\end{aligned}
$$

Let $0 \leq \rho \leq 1$, then

$$
\begin{aligned}
& \left(\rho h_{1}+(1-\rho) h_{2}\right)(t)=\sigma \beta \sum_{j=1}^{l_{2}} d_{j} \int_{0}^{\phi\left(\tau_{j}\right)} \frac{Q^{\prime}(s)\left(Q\left(\phi\left(\tau_{j}\right)\right)-Q(s)\right)^{\alpha-1}}{\Gamma(\alpha)} A(s) \eta(s) d s \\
& \quad+\sigma \beta \sum_{j=1}^{l_{2}} d_{j} \int_{0}^{\phi\left(\tau_{j}\right)} \frac{Q^{\prime}(s)\left(Q\left(\phi\left(\tau_{j}\right)\right)-Q(s)\right)^{\alpha-1}}{\Gamma(\alpha)}\left(\rho \psi_{1}(s)+(1-\rho) \psi_{2}(s)\right) d s \\
& \quad-\sigma \sum_{i=1}^{l_{1}} c_{i} \int_{0}^{\theta\left(t_{i}\right)} \frac{Q^{\prime}(s)\left(Q\left(\theta\left(t_{i}\right)\right)-Q(s)\right)^{\alpha-1}}{\Gamma(\alpha)} A(s) \eta(s) d s \\
& \quad-\sigma \sum_{i=1}^{l_{1}} c_{i} \int_{0}^{\theta\left(t_{i}\right)} \frac{Q^{\prime}(s)\left(Q\left(\theta\left(t_{i}\right)\right)-Q(s)\right)^{\alpha-1}}{\Gamma(\alpha)}\left(\rho \psi_{1}(s)+(1-\rho) \psi_{2}(s)\right) d s \\
& +\frac{1}{\Gamma(\alpha)} \int_{0}^{t} Q^{\prime}(s)(Q(t)-Q(s))^{\alpha-1} A(s) \eta(s) d s \\
& \quad+\frac{1}{\Gamma(\alpha)} \int_{0}^{t} Q^{\prime}(s)(Q(t)-Q(s))^{\alpha-1}\left(\rho \psi_{1}(s)+(1-\rho) \psi_{2}(s)\right) d s .
\end{aligned}
$$

From (H3), we have $F$ takes convex values. Hence, $\left(\rho h_{1}+(1-\rho) h_{2}\right) \in \Upsilon_{\eta}$. Thus, $S_{F, \eta}^{1}$ is convex.

Step 2. $\gamma$ is completely continuous. First, we will prove that $r$ is bounded. Let $r>0$. Define $B_{r}=\left\{\eta \in C(I, \mathbb{R}):\|\eta\|_{\infty} \leq r\right\}$ and let $\eta \in B_{r}$. From (H4), we have that

$$
\begin{aligned}
|h(t)| \leq \mid & \sigma \beta \sum_{j=1}^{l_{2}} d_{j} \int_{0}^{\tau_{j}} \frac{Q^{\prime}(s)\left(Q\left(\tau_{j}\right)-Q(s)\right)^{\alpha-1}}{\Gamma(\alpha)} A(s) \eta(s) d s \\
& +\sigma \beta \sum_{j=1}^{l_{2}} d_{j} \int_{0}^{\tau_{j}} \frac{Q^{\prime}(s)\left(Q\left(\tau_{j}\right)-Q(s)\right)^{\alpha-1}}{\Gamma(\alpha)} \psi(s) d s \\
& -\sigma \sum_{i=1}^{l_{1}} c_{i} \int_{0}^{t_{i}} \frac{Q^{\prime}(s)\left(Q\left(t_{i}\right)-Q(s)\right)^{\alpha-1}}{\Gamma(\alpha)} A(s) \eta(s) d s \\
& -\sigma \sum_{i=1}^{l_{1}} c_{i} \int_{0}^{t_{i}} \frac{Q^{\prime}(s)\left(Q\left(t_{i}\right)-Q(s)\right)^{\alpha-1}}{\Gamma(\alpha)} \psi(s) d s \\
& +\frac{1}{\Gamma(\alpha)} \int_{0}^{t} Q^{\prime}(s)(Q(t)-Q(s))^{\alpha-1} A(s) \eta(s) d s \\
& +\frac{1}{\Gamma(\alpha)} \int_{0}^{t} Q^{\prime}(s)(Q(t)-Q(s))^{\alpha-1} \psi(s) d s \mid,
\end{aligned}
$$

for all $t \in I$. Therefore, we get

$$
\begin{aligned}
\|h\|_{\infty} \leq & |\sigma \beta| \sum_{j=1}^{l_{2}} d_{j} \int_{0}^{\tau_{j}} \frac{Q^{\prime}(s)\left(Q\left(\tau_{j}\right)-Q(s)\right)^{\alpha-1}}{\Gamma(\alpha)}|A(s)|\|\eta\|_{\infty} d s \\
& +|\sigma \beta| K \sum_{j=1}^{l_{2}} d_{j} \int_{0}^{\tau_{j}} \frac{Q^{\prime}(s)\left(Q\left(\tau_{j}\right)-Q(s)\right)^{\alpha-1}}{\Gamma(\alpha)} p(s) d s \\
& +|\sigma| \sum_{i=1}^{l_{1}} c_{i} \int_{0}^{t_{i}} \frac{Q^{\prime}(s)\left(Q\left(t_{i}\right)-Q(s)\right)^{\alpha-1}}{\Gamma(\alpha)}|A(s)|\|\eta\|_{\infty} d s \\
& +|\sigma| K \sum_{i=1}^{l_{1}} c_{i} \int_{0}^{t_{i}} \frac{Q^{\prime}(s)\left(Q\left(t_{i}\right)-Q(s)\right)^{\alpha-1}}{\Gamma(\alpha)} p(s) d s \\
& +\frac{1}{\Gamma(\alpha)} \int_{0}^{t} Q^{\prime}(s)(Q(t)-Q(s))^{\alpha-1}|A(s)|\|\eta\|_{\infty} d s \\
& +\frac{K}{\Gamma(\alpha)} \int_{0}^{t} Q^{\prime}(s)(Q(t)-Q(s))^{\alpha-1} p(s) d s
\end{aligned}
$$




$$
\leq\left(L_{A} r \frac{(Q(T)-Q(0))^{\alpha}}{\Gamma(\alpha+1)}+K M\right)\left(|\sigma \beta| \sum_{j=1}^{l_{2}} d_{j}+|\sigma| \sum_{i=1}^{l_{1}} c_{i}+1\right) .
$$

Hence, $r$ sends bounded sets to bounded sets in $C(I, \mathbb{R})$. Secondly, we will prove $r$ sends $B_{r}$ into equicontinuous sets of $C(I, \mathbb{R})$. Let $t_{1}^{*}, t_{2}^{*} \in I$ such that $t_{1}^{*}<t_{2}^{*}$.

Then, for all $h \in \Upsilon_{\eta}$ and $\eta \in B_{r}$, we have

$$
\begin{aligned}
h\left(t_{2}^{*}\right)-h\left(t_{1}^{*}\right)= & \frac{1}{\Gamma(\alpha)} \int_{0}^{t_{2}^{*}} Q^{\prime}(s)\left(Q\left(t_{2}^{*}\right)-Q(s)\right)^{\alpha-1} A(s) \eta(s) d s \\
& -\frac{1}{\Gamma(\alpha)} \int_{0}^{t_{1}^{*}} Q^{\prime}(s)\left(Q\left(t_{1}^{*}\right)-Q(s)\right)^{\alpha-1} A(s) \eta(s) d s \\
& +\frac{1}{\Gamma(\alpha)} \int_{0}^{t_{2}^{*}} Q^{\prime}(s)\left(Q\left(t_{2}^{*}\right)-Q(s)\right)^{\alpha-1} \psi(s) d s \\
& -\frac{1}{\Gamma(\alpha)} \int_{0}^{t_{1}^{*}} Q^{\prime}(s)\left(Q\left(t_{1}^{*}\right)-Q(s)\right)^{\alpha-1} \psi(s) d s .
\end{aligned}
$$

Hence, we get

$$
\begin{aligned}
& \left|h\left(t_{2}^{*}\right)-h\left(t_{1}^{*}\right)\right| \leq \frac{1}{\Gamma(\alpha)} \int_{0}^{t_{1}^{*}} Q^{\prime}(s)\left[\left(Q\left(t_{2}^{*}\right)-Q(s)\right)^{\alpha-1}-\left(Q\left(t_{1}^{*}\right)-Q(s)\right)^{\alpha-1}\right] \\
& \cdot\left(|A(s)|\|\eta\|_{\infty}+\|\psi\|_{\infty}\right) d s+\frac{1}{\Gamma(\alpha)} \\
& \cdot \int_{t_{1}^{*}}^{t_{2}^{*}} Q^{\prime}(s)\left(Q\left(t_{2}^{*}\right)-Q(s)\right)^{\alpha-1}\left(|A(s)|\|\eta\|_{\infty}+\|\psi\|_{\infty}\right) d s .
\end{aligned}
$$

Next, by the Lagrange mean-value classical theorem, we obtain

$$
\begin{aligned}
\left|h\left(t_{2}^{*}\right)-h\left(t_{1}^{*}\right)\right| \leq & \frac{(\alpha-1)\left(t_{2}^{*}-t_{1}^{*}\right) Q^{\prime}(\widehat{t})}{\Gamma(\alpha)} \int_{0}^{t_{1}^{*}} Q^{\prime}(s) \\
& \cdot(Q(\widehat{t})-Q(s))^{\alpha-2}\left(L_{A} r+\|\psi\|_{\infty}\right) d s \\
& +\frac{1}{\Gamma(\alpha)} \int_{t_{1}^{*}}^{t_{2}^{*}} Q^{\prime}(s)\left(Q\left(t_{2}^{*}\right)-Q(s)\right)^{\alpha-1} \\
& \cdot\left(L_{A} r+\|\psi\|_{\infty}\right) d s,
\end{aligned}
$$

where $t_{1}^{*}<\hat{t}<t_{2}^{*}$. As $t_{1}^{*} \longrightarrow t_{2}^{*},\left|h\left(t_{2}^{*}\right)-h\left(t_{1}^{*}\right)\right| \longrightarrow 0$. Thus, $\Upsilon\left(B_{r}\right)$ is equicontinuous. From the Arzela-Ascoli theorem, we get $r$ is completely continuous.

Step 3. $r$ is u.s.c. We only need to show that $r$ has a closed graph to be u.s.c. Let $\eta_{n} \longrightarrow \widehat{\eta}$ and $h_{n} \longrightarrow \widehat{h}$ where $h_{n} \in Y \eta_{n}$. We need to show that $\widehat{h} \in Y \widehat{\eta}$. Associated with $h_{n}$ $\in \Upsilon_{\eta_{n}}$, there exists $\psi_{n} \in S_{F, \eta_{n}}^{1}$ such that for all $t \in I$, we have

$$
\begin{aligned}
h_{n}(t)= & \sigma \beta \sum_{j=1}^{l_{2}} d_{j} \int_{0}^{\phi\left(\tau_{j}\right)} \frac{Q^{\prime}(s)\left(Q\left(\phi\left(\tau_{j}\right)\right)-Q(s)\right)^{\alpha-1}}{\Gamma(\alpha)} A(s) \eta_{n}(s) d s \\
& +\sigma \beta \sum_{j=1}^{l_{2}} d_{j} \int_{0}^{\phi\left(\tau_{j}\right)} \frac{Q^{\prime}(s)\left(Q\left(\phi\left(\tau_{j}\right)\right)-Q(s)\right)^{\alpha-1}}{\Gamma(\alpha)} \psi_{n}(s) d s \\
& -\sigma \sum_{i=1}^{l_{1}} c_{i} \int_{0}^{\theta\left(t_{i}\right)} \frac{Q^{\prime}(s)\left(Q\left(\theta\left(t_{i}\right)\right)-Q(s)\right)^{\alpha-1}}{\Gamma(\alpha)} A(s) \eta_{n}(s) d s \\
& -\sigma \sum_{i=1}^{l_{1}} c_{i} \int_{0}^{\theta\left(t_{i}\right)} \frac{Q^{\prime}(s)\left(Q\left(\theta\left(t_{i}\right)\right)-Q(s)\right)^{\alpha-1}}{\Gamma(\alpha)} \psi_{n}(s) d s \\
& +\frac{1}{\Gamma(\alpha)} \int_{0}^{t} Q^{\prime}(s)(Q(t)-Q(s))^{\alpha-1} A(s) \eta_{n}(s) d s \\
& +\frac{1}{\Gamma(\alpha)} \int_{0}^{t} Q^{\prime}(s)(Q(t)-Q(s))^{\alpha-1} \psi_{n}(s) d s .
\end{aligned}
$$

We want to show that there exists $\widehat{\psi} \in S_{F, \widehat{\eta}}^{1}$ such that for each $t \in I$, we get

$$
\begin{aligned}
\widehat{h}(t)= & \sigma \beta \sum_{j=1}^{l_{2}} d_{j} \int_{0}^{\phi\left(\tau_{j}\right)} \frac{Q^{\prime}(s)\left(Q\left(\phi\left(\tau_{j}\right)\right)-Q(s)\right)^{\alpha-1}}{\Gamma(\alpha)} A(s) \widehat{\eta}(s) d s \\
& +\sigma \beta \sum_{j=1}^{l_{2}} d_{j} \int_{0}^{\phi\left(\tau_{j}\right)} \frac{Q^{\prime}(s)\left(Q\left(\phi\left(\tau_{j}\right)\right)-Q(s)\right)^{\alpha-1}}{\Gamma(\alpha)} \widehat{\psi}(s) d s \\
& -\sigma \sum_{i=1}^{l_{1}} c_{i} \int_{0}^{\theta\left(t_{i}\right)} \frac{Q^{\prime}(s)\left(Q\left(\theta\left(t_{i}\right)\right)-Q(s)\right)^{\alpha-1}}{\Gamma(\alpha)} A(s) \widehat{\eta}(s) d s \\
& -\sigma \sum_{i=1}^{l_{1}} c_{i} \int_{0}^{\theta\left(t_{i}\right)} \frac{Q^{\prime}(s)\left(Q\left(\theta\left(t_{i}\right)\right)-Q(s)\right)^{\alpha-1}}{\Gamma(\alpha)} \widehat{\psi}(s) d s \\
& +\frac{1}{\Gamma(\alpha)} \int_{0}^{t} Q^{\prime}(s)(Q(t)-Q(s))^{\alpha-1} A(s) \widehat{\eta}(s) d s \\
& +\frac{1}{\Gamma(\alpha)} \int_{0}^{t} Q^{\prime}(s)(Q(t)-Q(s))^{\alpha-1} \widehat{\psi}(s) d s .
\end{aligned}
$$

Define the linear continuous operator $\Theta: L^{1}(I, \mathbb{R}) \longrightarrow$ $C(I, \mathbb{R})$ by

$$
\begin{aligned}
\Theta(\psi)(t)= & \sigma \beta \sum_{j=1}^{l_{2}} d_{j} \int_{0}^{\phi\left(\tau_{j}\right)} \frac{Q^{\prime}(s)\left(Q\left(\phi\left(\tau_{j}\right)\right)-Q(s)\right)^{\alpha-1}}{\Gamma(\alpha)} A(s) \eta(s) d s \\
& +\sigma \beta \sum_{j=1}^{l_{2}} d_{j} \int_{0}^{\phi\left(\tau_{j}\right)} \frac{Q^{\prime}(s)\left(Q\left(\phi\left(\tau_{j}\right)\right)-Q(s)\right)^{\alpha-1}}{\Gamma(\alpha)} \psi(s) d s \\
& -\sigma \sum_{i=1}^{l_{1}} c_{i} \int_{0}^{\theta\left(t_{i}\right)} \frac{Q^{\prime}(s)\left(Q\left(\theta\left(t_{i}\right)\right)-Q(s)\right)^{\alpha-1}}{\Gamma(\alpha)} A(s) \eta(s) d s \\
& -\sigma \sum_{i=1}^{l_{1}} c_{i} \int_{0}^{\theta\left(t_{i}\right)} \frac{Q^{\prime}(s)\left(Q\left(\theta\left(t_{i}\right)\right)-Q(s)\right)^{\alpha-1}}{\Gamma(\alpha)} \psi(s) d s \\
& +\frac{1}{\Gamma(\alpha)} \int_{0}^{t} Q^{\prime}(s)(Q(t)-Q(s))^{\alpha-1} A(s) \eta(s) d s \\
& +\frac{1}{\Gamma(\alpha)} \int_{0}^{t} Q^{\prime}(s)(Q(t)-Q(s))^{\alpha-1} \psi(s) d s .
\end{aligned}
$$


Hence, we get

$$
\begin{aligned}
\left|h_{n}(t)-\widehat{h}(t)\right| \leq & |\sigma \beta| L_{A} \sum_{j=1}^{l_{2}} d_{j} \int_{0}^{\phi\left(\tau_{j}\right)} \frac{Q^{\prime}(s)\left(Q\left(\phi\left(\tau_{j}\right)\right)-Q(s)\right)^{\alpha-1}}{\Gamma(\alpha)}\left|\eta_{n}(s)-\widehat{\eta}(s)\right| d s \\
& +|\sigma \beta| \sum_{j=1}^{l_{2}} d_{j} \int_{0}^{\phi\left(\tau_{j}\right)} \frac{Q^{\prime}(s)\left(Q\left(\phi\left(\tau_{j}\right)\right)-Q(s)\right)^{\alpha-1}}{\Gamma(\alpha)}\left|\psi_{n}(s)-\widehat{\psi}(s)\right| d s \\
& +|\sigma| L_{A} \sum_{i=1}^{l_{1}} c_{i} \int_{0}^{\theta\left(t_{i}\right)} \frac{Q^{\prime}(s)\left(Q\left(\theta\left(t_{i}\right)\right)-Q(s)\right)^{\alpha-1}}{\Gamma(\alpha)}\left|\eta_{n}(s)-\widehat{\eta}(s)\right| d s \\
& +|\sigma| \sum_{i=1}^{l_{1}} c_{i} \int_{0}^{\theta\left(t_{i}\right)} \frac{Q^{\prime}(s)\left(Q\left(\theta\left(t_{i}\right)\right)-Q(s)\right)^{\alpha-1}}{\Gamma(\alpha)}\left|\psi_{n}(s)-\widehat{\psi}(s)\right| d s \\
& +\frac{L_{A}}{\Gamma(\alpha)} \int_{0}^{t} Q^{\prime}(s)(Q(t)-Q(s))^{\alpha-1}\left|\eta_{n}(s)-\widehat{\eta}(s)\right| d s \\
& +\frac{1}{\Gamma(\alpha)} \int_{0}^{t} Q^{\prime}(s)(Q(t)-Q(s))^{\alpha-1}\left|\psi_{n}(s)-\widehat{\psi}(s)\right| d s .
\end{aligned}
$$

Thus, $h_{n}(t) \longrightarrow \widehat{h}(t)$ as $n \longrightarrow \infty$. From Lemma 4, we can see $\Theta^{\circ} S_{F, \eta}^{1}$ is a closed graph in $C(I, \mathbb{R}) \times C(I, \mathbb{R})$ and $h_{n} \in$ $\Theta\left(S_{F, \eta_{n}}^{1}\right)$. Since $\eta_{n} \longrightarrow \widehat{\eta}$, then $\widehat{h}(t)$ satisfies equation (41) for some $\psi \in S_{F, \widehat{\eta}}^{1}$. Thus, $\gamma$ is an u.s.c.

Step 4. There exists an open set $\Omega \in C(I, \mathbb{R})$ such that $\eta \in \delta$ $\gamma \eta$ for some $\delta \in(0,1)$ and $\eta \in \partial \Omega$.

Let $\delta \in(0,1)$ and $\eta \in \delta \Upsilon \eta$. Then, there exists $\psi \in L^{1}(I, \mathbb{R})$ with $\psi \in S_{F, \eta}^{1}$ such that for all $t \in I$, we have

$$
\begin{aligned}
\eta(t)= & \delta\left[\sigma \beta \sum_{j=1}^{l_{2}} d_{j} \int_{0}^{\phi\left(\tau_{j}\right)} \frac{Q^{\prime}(s)\left(Q\left(\phi\left(\tau_{j}\right)\right)-Q(s)\right)^{\alpha-1}}{\Gamma(\alpha)} A(s) \eta(s) d s\right. \\
& +\sigma \beta \sum_{j=1}^{l_{2}} d_{j} \int_{0}^{\phi\left(\tau_{j}\right)} \frac{Q^{\prime}(s)\left(Q\left(\phi\left(\tau_{j}\right)\right)-Q(s)\right)^{\alpha-1}}{\Gamma(\alpha)} \psi(s) d s \\
& -\sigma \sum_{i=1}^{l_{1}} c_{i} \int_{0}^{\theta\left(t_{i}\right)} \frac{Q^{\prime}(s)\left(Q\left(\theta\left(t_{i}\right)\right)-Q(s)\right)^{\alpha-1}}{\Gamma(\alpha)} A(s) \eta(s) d s \\
& -\sigma \sum_{i=1}^{l_{1}} c_{i} \int_{0}^{\theta\left(t_{i}\right)} \frac{Q^{\prime}(s)\left(Q\left(\theta\left(t_{i}\right)\right)-Q(s)\right)^{\alpha-1}}{\Gamma(\alpha)} \psi(s) d s \\
& +\frac{1}{\Gamma(\alpha)} \int_{0}^{t} Q^{\prime}(s)(Q(t)-Q(s))^{\alpha-1} A(s) \eta(s) d s \\
& \left.+\frac{1}{\Gamma(\alpha)} \int_{0}^{t} Q^{\prime}(s)(Q(t)-Q(s))^{\alpha-1} \psi(s) d s\right] .
\end{aligned}
$$

As in the proof of Step 2, we get that

$$
\begin{aligned}
\|\eta\|_{\infty} \leq & \delta\left(L_{A}\|\eta\|_{\infty} \frac{(Q(T)-Q(0))^{\alpha}}{\Gamma(\alpha+1)}+K M\right) \\
& \cdot\left(|\sigma \beta| \sum_{j=1}^{l_{2}} d_{j}+|\sigma| \sum_{i=1}^{l_{1}} c_{i}+1\right) \\
\leq & \left(L_{A}\|\eta\|_{\infty} \frac{(Q(T)-Q(0))^{\alpha}}{\Gamma(\alpha+1)}+K M\right) \\
& \cdot\left(|\sigma \beta| \sum_{j=1}^{l_{2}} d_{j}+|\sigma| \sum_{i=1}^{l_{1}} c_{i}+1\right) .
\end{aligned}
$$

Hence, we get

$\frac{\Gamma(\alpha+1)\|\eta\|_{\infty}}{\left(L_{A}\|\eta\|_{\infty}(Q(T)-Q(0))^{\alpha}+K M \Gamma(\alpha+1)\right)\left(|\sigma \beta| \sum_{j=1}^{l_{2}} d_{j}+|\sigma| \sum_{i=1}^{l_{1}} c_{j}+1\right)} \leq 1$.

From (H5), there exists $M^{*}$ such that $M^{*} \neq\|\eta\|_{\infty}$. Let $\Omega=\left\{\eta \in C(I, \mathbb{R}):\|\eta\|_{\infty}<M^{*}+1\right\}$. Thus, there is no $\eta \in \partial \Omega$ such that $\eta \in \delta \Upsilon_{\eta}$ for $\delta \in(0,1)$. Hence, $\gamma: \bar{\Omega} \longrightarrow P_{c v p}\left(B_{r}\right)$ is u.s.c. From Theorem 5, we deduce that $r$ has a fixed point $\eta \in \bar{\Omega}$ which is a solution of the system (3).

Theorem 11. Assume that (H1)-(H4) hold. In addition, suppose

(H6) there exists a nondecreasing continuous function $\Xi:[0, \infty) \longrightarrow[0, \infty)$ and $q \in C\left(I, \mathbb{R}_{+}\right)$such that

$$
\|F(t, \eta(t))\|=\sup \{|\rho|: \rho \in W(t, \eta)\} \leq q(t) \Xi(|\rho|),
$$

for all $(t, \eta) \in I \times \mathbb{R}$, and there exists $\kappa>0$ such that

$$
\frac{\Gamma(\alpha+1) \kappa}{\left(L_{A} \kappa+\|q\|_{\infty} \Xi(\kappa)\right)(Q(T)-Q(0))^{\alpha}\left(|\sigma \beta| \sum_{j=1}^{l_{2}} d_{j}+|\sigma| \sum_{l=1}^{l_{l}} c_{i}+1\right)} \leq 1 .
$$

Then, the system (3) has at least one solution on I.

Proof. Define the multivalued operator $r: C(I, \mathbb{R}) \longrightarrow P(C$ $(I, \mathbb{R}))$ as in equation (31) of Theorem 10.

Step 1. $\Upsilon_{\eta}$ is convex for all $\eta \in C(I, \mathbb{R})$. By doing the same steps as in the proof of Theorem 10, we can get that $Y_{\eta}$ is convex for all $\eta \in C(I, \mathbb{R})$.

Step 2. $r$ is completely continuous. First, we will prove that $r$ is bounded. Let $r_{0}>0$. Define $B_{r_{0}}=\left\{\eta \in C(I, \mathbb{R}):\|\eta\|_{\infty} \leq r_{0}\right\}$ and let $\eta \in B_{r_{0}}$. From (H4), we have that

$$
\begin{aligned}
|h(t)| \leq \mid & \sigma \beta \sum_{j=1}^{l_{2}} d_{j} \int_{0}^{\tau_{j}} \frac{Q^{\prime}(s)\left(Q\left(\tau_{j}\right)-Q(s)\right)^{\alpha-1}}{\Gamma(\alpha)} A(s) \eta(s) d s \\
& +\sigma \beta \sum_{j=1}^{l_{2}} d_{j} \int_{0}^{\tau_{j}} \frac{Q^{\prime}(s)\left(Q\left(\tau_{j}\right)-Q(s)\right)^{\alpha-1}}{\Gamma(\alpha)} \psi(s) d s \\
& -\sigma \sum_{i=1}^{l_{1}} c_{i} \int_{0}^{t_{i}} \frac{Q^{\prime}(s)\left(Q\left(t_{i}\right)-Q(s)\right)^{\alpha-1}}{\Gamma(\alpha)} A(s) \eta(s) d s \\
& -\sigma \sum_{i=1}^{l_{1}} c_{i} \int_{0}^{t_{i}} \frac{Q^{\prime}(s)\left(Q\left(t_{i}\right)-Q(s)\right)^{\alpha-1}}{\Gamma(\alpha)} \psi(s) d s \\
& +\frac{1}{\Gamma(\alpha)} \int_{0}^{t} Q^{\prime}(s)(Q(t)-Q(s))^{\alpha-1} A(s) \eta(s) d s \\
& +\frac{1}{\Gamma(\alpha)} \int_{0}^{t} Q^{\prime}(s)(Q(t)-Q(s))^{\alpha-1} \psi(s) d s \mid,
\end{aligned}
$$


for all $t \in I$. Therefore, we get

$$
\begin{aligned}
\|h\|_{\infty} \leq & |\sigma \beta| \sum_{j=1}^{l_{2}} d_{j} \int_{0}^{\tau_{j}} \frac{Q^{\prime}(s)\left(Q\left(\tau_{j}\right)-Q(s)\right)^{\alpha-1}}{\Gamma(\alpha)}|A(s)|\|\eta\|_{\infty} d s \\
& +|\sigma \beta|\|q\|_{\infty} \Xi\left(r_{0}\right) \sum_{j=1}^{l_{2}} d_{j} \int_{0}^{\tau_{j}} \frac{Q^{\prime}(s)\left(Q\left(\tau_{j}\right)-Q(s)\right)^{\alpha-1}}{\Gamma(\alpha)} d s \\
& +|\sigma| \sum_{i=1}^{l_{1}} c_{i} \int_{0}^{t_{i}} \frac{Q^{\prime}(s)\left(Q\left(t_{i}\right)-Q(s)\right)^{\alpha-1}}{\Gamma(\alpha)}|A(s)|\|\eta\|_{\infty} d s \\
& +|\sigma|\|q\|_{\infty} \Xi\left(r_{0}\right) \sum_{i=1}^{l_{1}} c_{i} \int_{0}^{t_{i}} \frac{Q^{\prime}(s)\left(Q\left(\tau_{j}\right)-Q(s)\right)^{\alpha-1}}{\Gamma(\alpha)} d s \\
& +\frac{1}{\Gamma(\alpha)} \int_{0}^{t} Q^{\prime}(s)(Q(t)-Q(s))^{\alpha-1}|A(s)|\|\eta\|_{\infty} d s \\
& +\frac{\|q\|_{\infty} \Xi\left(r_{0}\right)}{\Gamma(\alpha)} \int_{0}^{t} Q^{\prime}(s)(Q(t)-Q(s))^{\alpha-1} d s \\
\leq & \left(\frac{L_{A} r_{0}+\|q\|_{\infty} \Xi\left(r_{0}\right)}{\Gamma(\alpha+1)}\right)(Q(T)-Q(0))^{\alpha} \\
& +\left(|\sigma \beta| \sum_{j=1}^{l_{2}} d_{j}+|\sigma| \sum_{i=1}^{l_{1}} c_{i}+1\right) .
\end{aligned}
$$

Hence, $r$ sends bounded sets to bounded sets in $C(I, R)$. Secondly, we will prove $Y$ sends $B_{r_{0}}$ into equicontinuous sets of $C(I, \mathbb{R})$. Let $t_{1}^{*}, t_{2}^{*} \in I$ such that $t_{1}^{*}<t_{2}^{*}$.

Then, for all $h \in \Upsilon_{\eta}$ and $\eta \in B_{r_{0}}$, we have

$$
\begin{aligned}
h\left(t_{2}^{*}\right)-h\left(t_{1}^{*}\right)= & \frac{1}{\Gamma(\alpha)} \int_{0}^{t_{2}^{*}} Q^{\prime}(s)\left(Q\left(t_{2}^{*}\right)-Q(s)\right)^{\alpha-1} A(s) \eta(s) d s \\
& -\frac{1}{\Gamma(\alpha)} \int_{0}^{t_{1}^{*}} Q^{\prime}(s)\left(Q\left(t_{1}^{*}\right)-Q(s)\right)^{\alpha-1} A(s) \eta(s) d s \\
& +\frac{1}{\Gamma(\alpha)} \int_{0}^{t_{2}^{*}} Q^{\prime}(s)\left(Q\left(t_{2}^{*}\right)-Q(s)\right)^{\alpha-1} \psi(s) d s \\
& -\frac{1}{\Gamma(\alpha)} \int_{0}^{t_{1}^{*}} Q^{\prime}(s)\left(Q\left(t_{1}^{*}\right)-Q(s)\right)^{\alpha-1} \psi(s) d s .
\end{aligned}
$$

Hence, we get

$$
\begin{aligned}
\left|h\left(t_{2}^{*}\right)-h\left(t_{1}^{*}\right)\right| \leq & \frac{\left(L_{A} r_{o}+\|q\|_{\infty} \Xi\left(r_{0}\right)\right)}{\Gamma(\alpha)} \int_{0}^{t_{1}^{*}} Q^{\prime}(s)\left|\left(Q\left(t_{2}^{*}\right)-Q(s)\right)^{\alpha-1}-\left(Q\left(t_{1}^{*}\right)-Q(s)\right)^{\alpha-1}\right| d s \\
& +\frac{\left(L_{A} r_{o}+\|q\|_{\infty} \Xi\left(r_{0}\right)\right)}{\Gamma(\alpha)} \int_{t_{1}^{2}}^{t_{i}} Q^{\prime}(s)\left(Q\left(t_{2}^{*}\right)-Q(s)\right)^{\alpha-1} d s \\
\leq & \frac{\left(L_{A} r_{o}+\|q\|_{\infty} \Xi\left(r_{0}\right)\right)}{\Gamma(\alpha)} \int_{0}^{t_{1}} Q^{\prime}(s)\left[\left(Q\left(t_{2}^{*}\right)-Q(s)\right)^{\alpha-1}-\left(Q\left(t_{1}^{*}\right)-Q(s)\right)^{\alpha-1}\right] d s \\
& +\frac{\left.\left(L_{A} r_{o}+\|q\|_{\infty} \Xi\left(r_{0}\right)\right)\right)}{\Gamma(\alpha)} \int_{t_{1}^{*}}^{t_{2}^{*}} Q^{\prime}(s)\left(Q\left(t_{2}^{*}\right)-Q(s)\right)^{\alpha-1} d s \\
\leq & \left.\frac{\left(L_{A} r_{o}+\|q\|_{\infty} \Xi\left(r_{0}\right)\right)}{\Gamma(\alpha+1)}\left[\left(Q\left(t_{2}^{*}\right)-Q(0)\right)^{\alpha}-\left(Q\left(t_{1}^{*}\right)-Q(0)\right)^{\alpha}\right)\right] .
\end{aligned}
$$

In view of continuity of $Q$, we have $\left|h\left(t_{2}^{*}\right)-h\left(t_{1}^{*}\right)\right| \longrightarrow 0$ as $t_{1}^{*} \longrightarrow t_{2}^{*}$. Thus, $r$ is completely continuous.

Step 3. $Y$ is u.s.c. As in the proof of Theorem 10, we have that $\gamma$ is an u.s.c.
Step 4. There exists an open set $\Omega \in C(I, \mathbb{R})$ such that $\eta / \delta \epsilon$ $\gamma_{\eta}$ for some $\delta \in(0,1)$ and $\eta \in \partial \Omega$.

Let $\delta \in(0,1)$ and $\eta \in \delta \Upsilon \eta$. Then, there exists $\psi \in L^{1}(I, \mathbb{R})$ with $\psi \in S_{F, \eta}^{1}$ such that for all $t \in I$, we have $\eta \in \delta Y \eta$ satisfies (44). As in the proof of Step 2, we have that

$$
\begin{aligned}
\|\eta\|_{\infty} \leq & \delta\left(\frac{L_{A}\|\eta\|_{\infty}+\|q\|_{\infty} \Xi\left(\|\eta\|_{\infty}\right)}{\Gamma(\alpha+1)}\right)(Q(T)-Q(0))^{\alpha} \\
& \cdot\left(|\sigma \beta| \sum_{j=1}^{l_{2}} d_{j}+|\sigma| \sum_{i=1}^{l_{1}} c_{i}+1\right) \\
\leq & \left(\frac{L_{A}\|\eta\|_{\infty}+\|q\|_{\infty} \Xi\left(\|\eta\|_{\infty}\right)}{\Gamma(\alpha+1)}\right)(Q(T)-Q(0))^{\alpha} \\
& \cdot\left(|\sigma \beta| \sum_{j=1}^{l_{2}} d_{j}+|\sigma| \sum_{i=1}^{l_{1}} c_{i}+1\right) .
\end{aligned}
$$

Hence, we get

$\frac{\Gamma(\alpha+1)\|\eta\|_{\infty}}{\left(L_{A}\|\eta\|_{\infty}+\|q\|_{\infty} \Xi\left(\|\eta\|_{\infty}\right)\right)(Q(T)-Q(0))^{\alpha}\left(|\sigma \beta| \sum_{j=1}^{l_{2}} d_{j}+|\sigma| \sum_{1=1}^{l_{1}} c_{i}+1\right)} \leq 1$.

From (H6), there exists $\kappa$ such that $\kappa \neq\|\eta\|_{\infty}$. Let $\Lambda=\{\eta$ $\left.\in C(I, \mathbb{R}):\|\eta\|_{\infty}<\kappa+1\right\}$. Thus, there is no $\eta \in \partial \Lambda$ such that $\eta \in \delta \Upsilon_{\eta}$ for $\delta \in(0,1)$. Hence, $Y: \bar{\Lambda} \longrightarrow P_{c v p}\left(B_{r_{0}}\right)$ is u.s.c. From Theorem 5, we deduce that $Y$ has a fixed point $\eta \in \bar{\Lambda}$ which is a solution of the system (3).

Putting $Q(t)=t$ in (3), we have the following result.

Corollary 12. Assume that (H1)-(H4) hold. In addition, suppose

(H7) there exists a nondecreasing continuous function $\Xi:[0, \infty) \longrightarrow[0, \infty)$ and $q \in C\left(I, \mathbb{R}_{+}\right)$such that

$$
\|F(t, \eta(t))\|=\sup \{|\rho|: \rho \in W(t, \eta)\} \leq q(t) \Xi(|\rho|),
$$

for all $(t, \eta) \in I \times \mathbb{R}$, and there exists $\kappa^{*}>0$ such that

$$
\frac{\Gamma(\alpha+1) \kappa^{*}}{\left(L_{A} \kappa^{*}+\|q\|_{\infty} \Xi\left(\kappa^{*}\right)\right) T^{\alpha}\left(|\sigma \beta| \sum_{j=1}^{l_{2}} d_{j}+|\sigma| \sum_{i=1}^{l_{1}} c_{i}+1\right)} \leq 1 .
$$

Then the system (2) has at least one solution on I.

Choosing $Q(t)=\ln (t)$ in system (3) and changing the interval $I$ with the interval $I_{e}=[1, e]$ in (3) and in all conditions (H1)-(H4), we obtain the following Caputo-Hadamard 
fractional system

$$
\left\{\begin{array}{l}
{ }^{C-H} D_{1^{+}}^{\alpha} \eta(t) \in A(t) \eta(t)+F(t, \eta(t)), \quad \text { a.e. } \quad t \in I_{e}=[1, e], \\
\sum_{i=1}^{l_{1}} c_{i} \eta\left(\theta\left(t_{i}\right)\right)=\beta \sum_{j=1}^{l_{2}} d_{j} \eta\left(\phi\left(\tau_{i}\right)\right), c_{i}, d_{j}>0, t_{i}, \tau_{j} \in I_{e}, \forall i=1, \cdots, l_{1}, j=1, \cdots, l_{2} .
\end{array}\right.
$$

The following result is a direct consequence of Theorem 11.

Corollary 13. Assume that (H1)-(H4) hold on $I_{e}$. In addition, suppose

(H8) there exists a nondecreasing continuous function $\Xi:[0, \infty) \longrightarrow[0, \infty)$ and $q \in C\left(I_{e}, \mathbb{R}_{+}\right)$such that

$$
\|F(t, \eta(t))\|=\sup \{|\rho|: \rho \in W(t, \eta)\} \leq q(t) \Xi(|\rho|) \text {, }
$$

for all $(t, \eta) \in I_{e} \times \mathbb{R}$, and there exists $\widehat{\kappa}>0$ such that

$$
\frac{\Gamma(\alpha+1) \widehat{\kappa}}{\left(L_{A} \widehat{\kappa}+\|q\|_{\infty} \Xi(\widehat{\kappa})\right)\left(|\sigma \beta| \sum_{j=1}^{l_{2}} d_{j}+|\sigma| \sum_{i=1}^{l_{1}} c_{i}+1\right)} \leq 1 .
$$

Then, the system (57) has at least one solution on $I_{e}$.

\section{Applications}

In the following examples, we point to how applied the abstract results in particular systems.

Example 14. Consider the following generalized fractional differential inclusion

$$
\left\{\begin{array}{l}
{ }^{*} D_{0^{+}, Q}^{\frac{2}{5}} \eta(t)-\frac{\cos (\eta(t))}{t^{2}+100} \in\left[0, \frac{|\eta(t)|}{|\eta(t)|+t^{2}+1}+3 t^{2}+6 t^{4}\right], \quad \text { a.e. } \quad t \in[0,1], \\
\sum_{i=1}^{2} 4^{i} \eta\left(t_{i}^{4}\right)=\frac{9}{4} \sum_{j=1}^{3} 3^{-j} \eta\left(\left(\tau_{j}\right)^{0.25}\right), t_{i}, \tau_{j} \in[0,1], \forall_{i}=1,2 ; j=1,2,3 .
\end{array}\right.
$$

Let $Q(t)=\left(t^{2}+t\right) / 2$. Here, we get $\alpha=2 / 5$,

$$
\begin{aligned}
& A(t) \eta(t)=\frac{\cos (\eta(t))}{t^{2}+100}, \\
& F(t, \eta)=\left[0, \frac{|\eta(t)|}{|\eta(t)|+t^{2}+1}+3 t^{2}+6 t^{4}\right], \\
& \theta:[0,1] \longrightarrow[0,1], \theta(t)=t^{4}, \\
& \phi:[0,1] \longrightarrow[0,1], \phi(t)=t^{0.25}
\end{aligned}
$$

From given information, we obtain that $L_{A}=0.01, T$ $=1, l_{1}=2, l_{2}=3, \sum_{i=1}^{2} 4^{i}=5 / 16, \sum_{j=1}^{3} 3^{-j}=7 / 8, \beta=2.25$. Hence, $\sigma=-0.6037735849$. In addition, it has $\|F(t, \eta)\| \leq 10$. Thus, $p(t)=1, K=10$ and $M=1.1273957159$. Then, there exists a constant $M^{*}$ such that $M^{*} \in(25.53, \infty)$ satisfying the inequality of (H5).

By Theorem 10, we know the system (60) has at least one solution.
Example 15. Consider the following generalized fractional differential inclusion

$$
\left\{\begin{array}{l}
{ }^{*} D_{0^{+}, Q}^{\frac{2}{3}} \eta(t)-\frac{\cos (\eta(t))}{t^{2}+100} \in\left[0, \frac{t|\eta(t)|}{t+1}\right], \quad \text { a.e. } t \in[0,1], \\
\sum_{i=4}^{2} 4^{i} \eta\left(t_{i}^{4}\right)=\frac{9}{4} \sum_{j=1}^{3} 3^{-j} \eta\left(\left(\tau_{j}\right)^{0.25}\right), t_{i}, \tau_{j} \in[0,1], \forall i=1,2 ; j=1,2,3 .
\end{array}\right.
$$

Let $Q(t)=\sqrt{t+1}$. Here, we get $\alpha=2 / 3$,

$$
\begin{gathered}
A(t) \eta(t)=\frac{\cos (n(t))}{t^{2}+100} \\
F(t, \eta)=\left[0, \frac{t|\eta(t)|}{t+1}\right], \\
\theta:[0,1] \longrightarrow[0,1], \theta(t)=t^{4}, \\
\phi:[0,1] \longrightarrow[0,1], \phi(t)=t^{0.25} .
\end{gathered}
$$

Thus, $L_{A}=0.01, T=1, l_{1}=2, l_{2}=3, \sum_{i=1}^{2} 4^{i}=5 / 16, \sum_{j=1}^{3}$ $3^{-j}=7 / 8$, and $\beta=2.25$. Therefore, $\sigma=-0.6037735849$. In addition, it has $\|F(t, \eta)\| \leq(t / t+1)|\eta(t)|$ Further, $q(t)=t$ / $(t+1),\|q\|_{\infty}=1$, and $\Xi\left(\|\eta\|_{\infty}\right)=\|\eta\|_{\infty}$. The condition (H6) of Theorem 11 is satisfied with $\kappa \in(0.5558151022, \infty)$. Consequently, all the hypotheses of Theorem 11 are satisfied. Thus, the problem (62) has at least one solution.

\section{Conclusions}

In this paper, we established the solvability of fractional differential inclusions involving the generalized Caputo operator by applying Leray-Schauder's alternative approach with the help of the Lagrange mean-value classical theorem. The proposed system studied in the present work is more practical and more generalized. The results given in this paper extended and developed some previous works. We presented some examples to illustrate the solvability results.

\section{Data Availability}

No data were used to support this study.

\section{Conflicts of Interest}

The authors declare that they have no conflicts of interest.

\section{References}

[1] I. Podlubny, Fractional Differential Equations: An Introduction to Fractional Derivatives, Fractional Differential Equations, Some Methods of Their Solution and Some of Their Applications, Academic Press, New York, 1999.

[2] R. L. Magin, Fractional Calculus in Bioengineering, Begell House Inc, USA, 2006.

[3] Y. Yu, P. Perdikaris, and G. E. Karniadakis, "Fractional modeling of viscoelasticity in 3D cerebral arteries and aneurysms," 
Journal of Computational Physics, vol. 323, article 219242, 2016.

[4] A. H. Hadian-Rasanan, D. Rahmati, S. Gorgin, and K. Parand, "A single layer fractional orthogonal neural network for solving various types of Lane Emden equation," New Astronomy, vol. 75, article 101307, 2020.

[5] P. Wu and H. Zhao, "Dynamics of an HIV infection model with two infection routes and evolutionary competition between two viral strains," Applied Mathematical Modelling, vol. 84, pp. 240-264, 2020.

[6] V.E. Tarasov, "Fractional Dynamics: Application of Fractional Calculus to Dynamics of Particles, Fields and Media, Springer, Heidelberg, Higher Education Press," Beijing, 2010.

[7] M. Benaim, J. Hofbaue, and S. Sorin, "Stochastic approximations and differential inclusions," SIAM Journal on Control and Optimization, vol. 44, no. 1, pp. 328-348, 2005.

[8] A. M. A. El-sayed, E. M. Hamdallah, and K. W. Elkadeky, "Solutions of a class of deviated-advanced nonlocal problems for the differential Inclusion $\mathrm{x}^{\prime}(\mathrm{t}) \in \mathrm{F}(\mathrm{t}, \mathrm{x}(\mathrm{t}))$," Abstract and Applied Analysis, vol. 2011, 9 pages, 2011.

[9] J. Wanga, A. G. Ibrahim, and M. Feckan, "Nonlocal impulsive fractional differential inclusions with fractional sectorial operators on Banach spaces," Applied Mathematics and Computation, vol. 257, pp. 103-118, 2015.

[10] T. Lian, C. Xue, and S. Deng, "Mild solutions to fractional differential inclusions with nonlocal conditions," Boundary Value Problems, vol. 2016, no. 1, 2016.

[11] C. Castaing, C. Godet-Thobie, P. D. Phung, and L. X. Truong, "On fractional differential inclusions with nonlocal boundary conditions," Fractional Calculus and Applied Analysis, vol. 22, no. 2, pp. 444-478, 2019.

[12] R. Almeida, "A Caputo fractional derivative of a function with respect to another function," Communications in Nonlinear Science and Numerical Simulation, vol. 44, pp. 460-481, 2017.

[13] R. Almeida, A. B. Malinowska, and M. T. Monterio, "Fractional differential equations with a Caputo derivative with respect to a kernel function and their applications," Mathematical Methods in the Applied Sciences, vol. 41, no. 1, pp. 336-352, 2018.

[14] F. Jarad and T. Abdeljawad, "Generalized fractional derivatives and Laplace transform," Discrete and Continuous Dynamical Systems - S, vol. 13, no. 3, pp. 709-722, 2020.

[15] C. Promsakon, E. Suntonsinsoungvon, S. K. Ntouyas, and J. Tariboon, "Impulsive boundary value problems containing Caputo fractional derivative of a function with respect to another function," Advances in Difference Equations, vol. 2019, no. 1, 2019.

[16] S. Belmor, F. Jarad, T. Abdeljawad, and M. A. Alqudah, "On fractional differential inclusion problems involving fractional order derivative with respect to another function," Fractals, vol. 28, p. 2040002, 2020.

[17] R. Almeida, "Fractional differential equations with mixed boundary conditions," Bulletin of the Malaysian Mathematical Sciences Society, vol. 42, no. 4, pp. 1687-1697, 2019.

[18] F. Jarad, S. Harikrishnan, K. Shah, and K. Kanagarajan, "Existence and stability results to a class of fractional random implicit differential equations involving a generalized Hilfer fractional derivative," Discrete \& Continuous Dynamical Systems-S, vol. 13, no. 3, pp. 723-739, 2020.

[19] D. Luo and Z. Luo, "Existence of solutions for fractional differential inclusions with initial value condition and non-instantaneous impulses," Univerzitet u Nišu, vol. 33, no. 17, pp. 5499-5510, 2019.

[20] B. Samet and H. Aydi, "Lyapunov-type inequalities for an antiperiodic fractional boundary value problem involving $\psi$-Caputo fractional derivative," Journal of inequalities and applications, vol. 2018, no. 1, 2018.

[21] M. A. E. Herzallah and A. H. A. Radwan, "Existence of mild solutions to semilinear fractional differential inclusion with deviated advanced nonlocal conditions," Journal of the Egyptian Mathematical Society, vol. 27, no. 1, 2019.

[22] J. R. Graef, J. Henderson, and A. Ouahab, Topological Methods for Differential Equations and Inclusions, Taylor and Francis, CRC Press, Boca Raton, FL, USA, 2019.

[23] A. Granas and J. Dugundji, Fixed Point Theory, Springer-Verlag, New York, 2005.

[24] Y. Luo and W. Wang, "Existence results for impulsive differential inclusions with nonlocal conditions," Journal of Fixed Point Theory and Applications, vol. 20, no. 2, 2018.

[25] R. P. Agarwal, U. Aksoy, E. Karapinar, and I. M. Erhan, "F-contraction mappings on metric-like spaces in connection with integral equations on time scales," RACSAM, vol. 114, no. 3, article 147, 2020.

[26] U. Aksoy, E. Karapinar, I. M. Erhan, and V. Rakočević, "MeirKeeler type contractions on modular metric spaces," Univerzitet $u$ Nišu, vol. 32, no. 10, pp. 3697-3707, 2018.

[27] U. Aksoy, E. Karapinar, and I. M. Erhan, "Fixed point theorems in complete modular metric spaces and an application to anti-periodic boundary value problems," Univerzitet $u$ Nišu, vol. 31, no. 17, pp. 5475-5488, 2017.

[28] U. Aksoy, E. Karapinar, and I. M. Erhan, "Fixed points of generalized alpha-admissible contractions on bmetric spaces with an application to boundary value problems," Journal of Nonlinear and Convex Analysis, vol. 17, pp. 1095-1108, 2016.

[29] E. Karapinar, I. M. Erhan, and U. Aksoy, "Weak $\psi$-contractions on partially ordered metric spaces and applications to boundary value problems," Boundary Value Problems, vol. 2014, Article ID 149, 2014.

[30] D. O'Regan, "Nonlinear alternatives for multivalued maps with applications to operator inclusions in abstract spaces," Proceedings of the American Mathematical Society, vol. 127, no. 12, pp. 3557-3564, 1999.

[31] A. A. Kilbas, H. M. Srivastava, and J. J. Trujillo, Theory and Applications of the Fractional Differential Equations, NorthHolland Mathematics studies, Elsevier, Amsterdam, 2006.

[32] R. Zafar, M. U. Rehman, and M. Shams, “On Caputo modification of Hadamard-type fractional derivative and fractional Taylor series," Advances in Difference Equations, vol. 2020, Article ID 219, 2020. 